\title{
Refractive index sensor based on an abrupt taper Michelson interferometer in a single-mode fiber
}

\author{
Zhaobing Tian, ${ }^{1, *}$ Scott S-H. Yam, ${ }^{2}$ and Hans-Peter Loock ${ }^{3}$ \\ ${ }^{1}$ Department of Physics, Engineering Physics, and Astronomy, Queen's University, Kingston, Ontario, K7L 3N6, \\ Canada \\ ${ }^{2}$ Department of Electrical and Computer Engineering, Queen's University, Kingston, Ontario, K7L 3N6, Canada \\ ${ }^{3}$ Department of Chemistry, Queen's University, Kingston, Ontario, K7L 3N6, Canada \\ *Corresponding author: tianzhaobing@gmail.com
}

Received February 28, 2008; revised April 9, 2008; accepted April 10, 2008; posted April 14, 2008 (Doc. ID 93286); published May 13, 2008

A simple refractive index sensor based on a Michelson interferometer in a single-mode fiber is constructed and demonstrated. The sensor consists of a single symmetrically abrupt taper region in a short piece of single-mode fiber that is terminated by $\sim 500 \mathrm{~nm}$ thick gold coating. The sensitivity of the new sensor is similar to that of a long-period-grating-type sensor, and its ease of fabrication offers a low-cost alternative to current sensing applications. (C) 2008 Optical Society of America

OCIS codes: $060.2370,120.3180,130.3120$.

Fiber Michelson interferometers are based on the general principle of superimposing two optical signals at the same wavelength but with a relative phase difference that is induced through travel in different media or along different path lengths [1]. To realize a Michelson interferometer in a single fiber device, one guided mode typically propagates in the optical fiber core, while the other is confined for a short length in the cladding or another core. To date, different approaches have been proposed to generate the second mode: namely, long period gratings (LPGs) [2], tilted fiber Bragg gratings [3], or coupling from a single-core fiber to a multicore fiber [4]. However, the manufacture of grating-type sensors requires precise and expensive phase masks, stringent photolithographic procedures, and photosensitized fiber. Coupling from the single-core to the multicore fiber also requires special fiber, and the method cannot be used for refractive index (RI) sensing applications since the two modes confined in the cores are not sensitive to the outer environment RI change.

In this Letter, a new compact, single taper-based Michelson interferometric sensor is described that can be made using standard equipment, such as a fusion splicer, and a standard single-mode fiber (SMF, such as Corning SMF-28). The device has a minimum insertion loss of $1 \mathrm{~dB}$ and a maximum interference extinction ratio of over $13 \mathrm{~dB}$. The sensitivity of the one-taper sensor to surrounding RI change was found to be comparable to that of similar structures made from identical LPGs, but its ease of fabrication offers a low-cost alternative in some RI sensing applications.

Taper structures of optical fibers have been extensively studied as power couplers, sensors, and adddrop multiplexers [5-7]. Previous work on power coupling and filtering focused largely on reducing the insertion loss by gradually tapering SMF to a waist diameter of several micrometers under high heat conditions. Hence, for such applications more gradual tapers or smaller taper angles are preferred. For larger taper angle or abrupt tapers light in the core will be partially coupled into the cladding and gradually attenuated. However, if a mirror, here a layer of gold coating on the fiber end facet, follows within centimeters $(L)$ of the taper, the attenuation of the cladding mode is negligible. At the mirror the cladding mode is reflected and coupled into counterpropagating cladding modes before being coupled back into the core at the taper section [8]. Owing to the resulting phase difference $\Phi$ between the core and cladding modes, a Michelson interferometer is created. The phase shift $\Phi$ can be approximated as [9]

$$
\Phi=4 \pi \Delta n_{\mathrm{eff}} L / \lambda,
$$

where $\Delta n_{\text {eff }}$ is the effective RI difference between the core and cladding modes and $\lambda$ is the input wavelength in vacuum.

When light emitted by a broadband source (BBS) propagates through this device the resulting interference spectrum can be recorded by an optical spectrum analyzer (OSA). The attenuation maxima are at wavelengths

$$
\lambda_{m}=4 \pi \Delta n_{\mathrm{eff}} L /(2 m+1) \pi,
$$

where $m$ is an integer.

If the RI of the environment surrounding the SMF is increased, for example, by submersing the section between the taper and mirror in distilled water, the effective RI of the cladding mode increases by $\delta n_{\text {eff }}$. Since the RI of the core mode stays almost constant

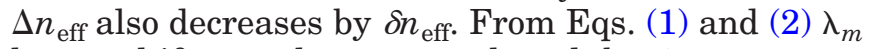
has to shift to a shorter wavelength by $\delta \lambda_{m}$.

$$
\delta \lambda_{m} \approx 4 \pi L \delta n_{\text {eff. }}
$$

From a measurement of $\delta \lambda_{m}$ one can either measure the RI of an unknown sample, or determine the concentration of a known solution sample. 
As shown in Fig. 1, light from a BBS (JDS Uniphase, $1530-1600 \mathrm{~nm}$ ) propagated through a circulator, then through a piece of SMF (without taper), was reflected back by the gold coating layer through the SMF and circulator again, and was finally collected by an OSA (ANDO, AQ6317B) and recorded as reference. The gold mirror was prepared by cleaving the SMF and depositing a gold layer $(\sim 500 \mathrm{~nm})$ with a sputter source. Such a source can simultaneously process thousands of fiber samples. A fusion splicer (Ericsson, model FSU 995FA) with a built-in taper program was used to fabricate the $3 \mathrm{~dB}$ taper. Figure 2 shows the image of one $3 \mathrm{~dB}$ taper taken by a CCD camera (Nikon) through a trinocular microscope. The length of the tapered region was measured as $734 \mu \mathrm{m}$, while the waist diameter was $40 \mu \mathrm{m}$. In [10], it was found that transmission spectrum of one taper is flat at $3 \mathrm{~dB}$ across the wavelength window. The fiber's protective coating layer was removed between the taper and the tip. The attenuation spectrum of the device (Fig. 3) shows a strong interference pattern and was obtained by subtracting the reference spectrum from the transmission spectrum of the device. The minimum attenuation of the interferometer is only $1 \mathrm{~dB}$, while the extinction ratio is larger than $13 \mathrm{~dB}$.

The Michelson interferometer was then mounted on a fiber holder and immersed in distilled water at different depths. As shown in Fig. 4, the attenuation maxima $\lambda_{m}$ are shifted to a shorter wavelength linearly with increasing immersion length. Since the RI

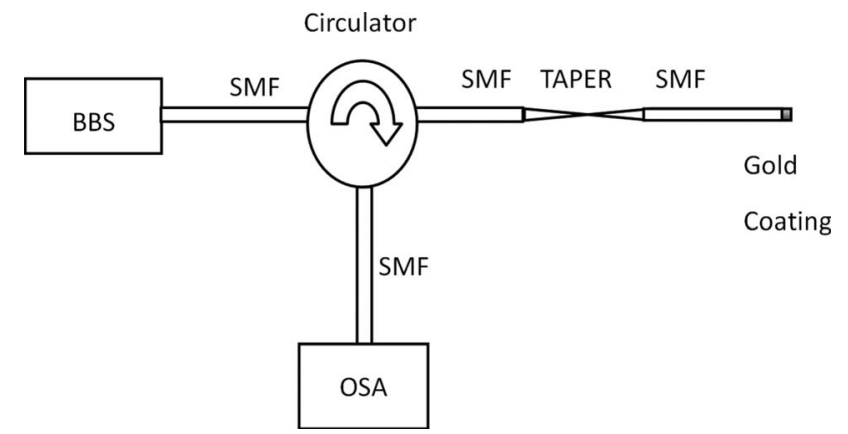

Fig. 1. Taper-based SMF Michelson interferometer.

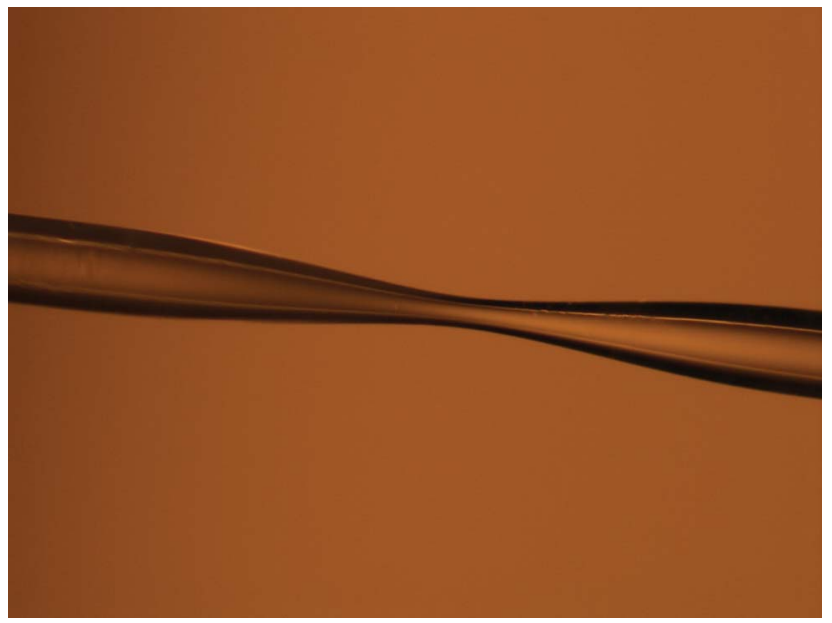

Fig. 2. (Color online) One $3 \mathrm{~dB}$ taper made by a fusion splicer.

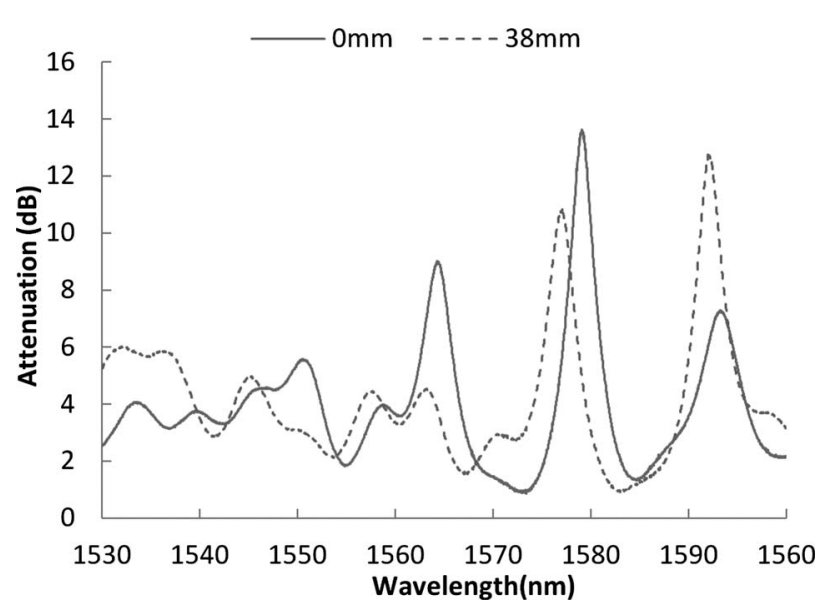

Fig. 3. Spectrum of Michelson interferometer in air $(0 \mathrm{~mm})$ and distilled water $(38 \mathrm{~mm})$.

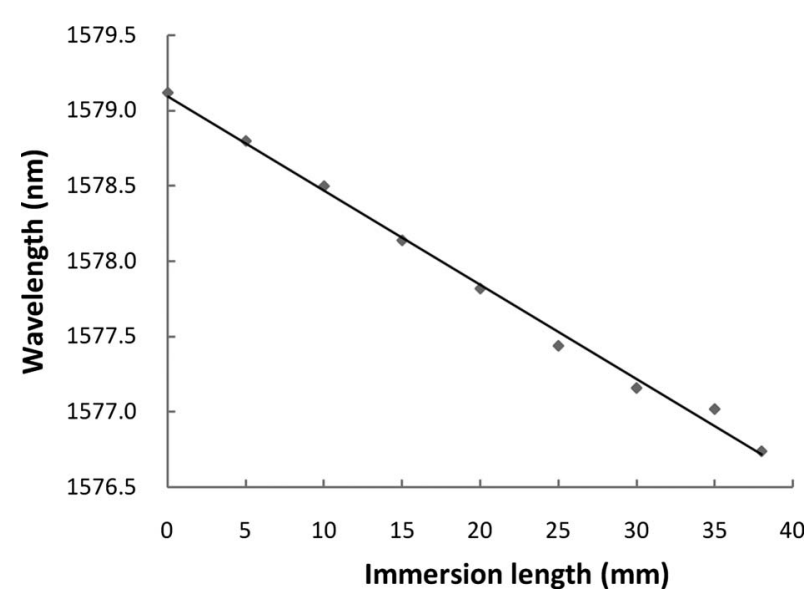

Fig. 4. Wavelength, $\lambda_{m}$, shift owing to the different immersion length in distilled water.

of the distilled water $(n=1.315$ at $1550 \mathrm{~nm})$ is larger than that of air (1.00), $\Delta n_{\text {eff }}$ becomes smaller with increasing immersion depth and, from Eqs. (1) and (3), the resulting peak attenuation wavelength is expected to move to a shorter wavelength. The dashed curve in Fig. 3 represents the spectrum of the device when immersed by $38 \mathrm{~mm}$ into distilled water. To determine the sensitivity with which the RI of an unknown solution can be obtained we prepared nine demethyl sulfoxide (DMSO)-water solutions with different volume concentrations $(0.0 \%, 4.0 \%, 8.0 \%$, $12.0 \%, 16.0 \%, 20.0 \%, 24.0 \%, 28.0 \%$, and $32.0 \%)$. The corresponding RIs (calculated from the Fresnel reflection using water as reference at $1550 \mathrm{~nm}$ and $25^{\circ} \mathrm{C}$ ) are $1.315,1.3208,1.3266,1.3324,1.3382$, $1.3441,1.35,1.3559$, and 1.3618 , respectively. Figure 5 shows the corresponding $\lambda_{m}$ shift. A change of RI by 0.01 causes a wavelength shift of $\sim 0.29 \mathrm{~nm}$ for an interaction length of $38 \mathrm{~mm}$. In a previous study [11] it was reported that for the same RI change a long period grating pair Mach-Zehnder interferometer sensor with an interaction length of $62 \mathrm{~mm}$ (which includes the length of the two gratings) causes a wavelength shift of $0.259 \mathrm{~nm}$. Given that the minimum wavelength resolution of the OSA is $15 \mathrm{pm}$, the 


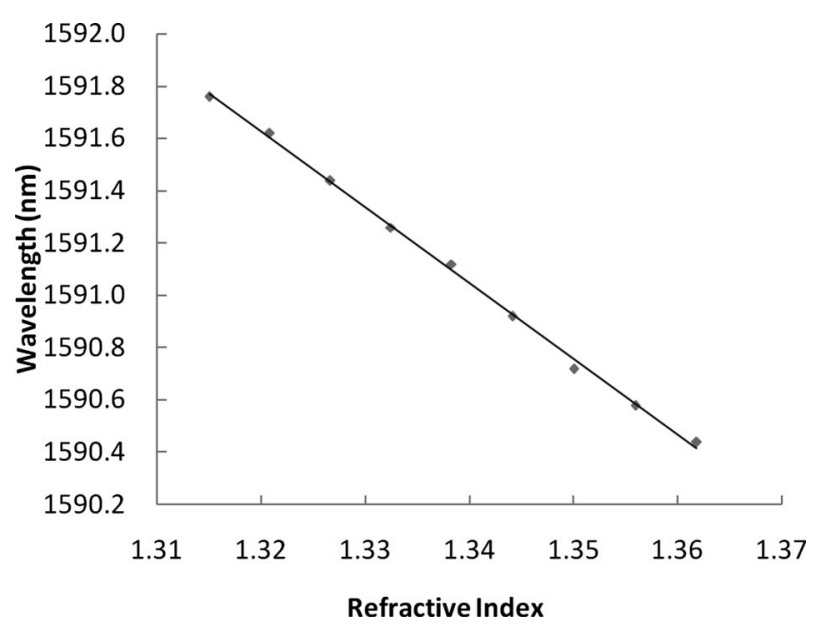

Fig. 5. Wavelength, $\lambda_{m}$, shift owing to different RI of a series of DMSO-water solutions.

RI differences that could be figured out are 5.1 $\times 10^{-4}$ and $5.8 \times 10^{-4}$ for the Michelson and MachZehnder interferometer sensors, respectively. The proposed sensor is more compact than the current Mach-Zehnder-based interferometers and can be made more quickly and at much lower cost. The fabrication of a taper structure is much simpler compared to that of a LPG pair, normally taking less than $1 \mathrm{~min}$. Also, normal transmission fiber Corning SMF-28 was used here to make abrupt tapers, whereas more expensive photosensitized fiber is needed to produce LPGs [11]. For the typical range of protein analytes (RI from 1.3 to 1.4 ), the response of the sensor should be linear as indicated by the straight line in Fig. 5. Also, based on Eq. (3) and Fig. 4 , increasing the interference arm of the device, i.e., the segment between the taper and mirror, linearly increases the sensitivity. The sensitivity can also be increased by using smaller diameter cladding SMF, which increases $\delta n_{\text {eff. }}$
In conclusion, a compact and stable single-taper Michelson interferometer RI sensor has been realized. The sensor demonstrated comparable sensitivity to that of a LPG pair Mach-Zehnder sensor, but is made in a much simpler fabrication process. This novel, low-cost interferometer may find application not only in chemical sensing but also in stress and temperature monitoring.

This work was supported by the Natural Science and Engineering Council of Canada (RGPIN 311817 06), Canadian Institute for Photonic Innovations, and Photonics Research Ontario. The authors acknowledge the Canadian Microelectronics Corporation for assistance in preparing the experiments. Some measurements were made with equipment from the National Microelectronics and Photonics Testing Collaboratory.

\section{References}

1. P. L. Swart, Meas. Sci. Technol. 15, 1576 (2004).

2. H. J. Patrick, A. D. Kersey, and F. Bucholtz, J. Lightwave Technol. 16, 1606 (1998).

3. G. Laffont and P. Ferdinand, Meas. Sci. Technol. 12, 765 (2001).

4. L. Yuan, J. Yang, Z. Liu, and J. Sun, Opt. Lett. 13, 2692 (2006).

5. J. D. Love, W. M. Henry, W. J. Stewart, R. J. Black, S. Lacroix, and F. Gonthier, IEE Proc. 138, 343 (1991).

6. B. E. Little, J. P. Laine, and H. A. Haus, J. Lightwave Technol. 17, 704 (1999).

7. Y. He and F. G. Shi, Opt. Commun. 260, 127 (2006).

8. O. Frazão, P. Caldas, F. M. Araújo, L. A. Ferreira, and J. L. Santos, Opt. Lett. 32, 1974 (2007).

9. T. Allsop, R. Reeves, D. J. Webb, I. Bennion, and R. Neal, Rev. Sci. Instrum. 73, 1702 (2002).

10. Z. Tian, S. S.-H. Yam, J. Barnes, W. Bock, P. Greig, J. M. Fraser, H. P. Loock, and R. D. Oleschuk, IEEE Photon. Technol. Lett. 20, 626 (2008).

11. J. F. Ding, A. P. Zhang, L. Y. Shao, J. H. Yan, and S. He, IEEE Photon. Technol. Lett. 17, 1247 (2005). 